\title{
The Genus Rosmarinus
}

Author(s): W. B. Turrill

Source: Bulletin of Miscellaneous Information (Royal Botanic Gardens, Kew), Vol. 1920, No. 3 ( 1920), pp. 105-108

Published by: Springer on behalf of Royal Botanic Gardens, Kew

Stable URL: http://www.jstor.org/stable/4120223

Accessed: 11-11-2015 03:40 UTC

Your use of the JSTOR archive indicates your acceptance of the Terms \& Conditions of Use, available at http://www.jstor.org/page/ info/about/policies/terms.jsp

JSTOR is a not-for-profit service that helps scholars, researchers, and students discover, use, and build upon a wide range of content in a trusted digital archive. We use information technology and tools to increase productivity and facilitate new forms of scholarship. For more information about JSTOR, please contact support@jstor.org. 
value of Vallonea, which at one time varied between $£ 20$ and $£ 25$ per ton, fell to such an extent during the years before the war that export-from Patras, at any rate, had almost ceased, but during the war the price rose again.

Lobel, loc. cit., fancied that he had seen the Vallonea oak in central Italy; "hujus speciei observasse memini publica via qua itur Pesaro Romam." His memory deceived him; he can only have seen the Turkey oak there (or possibly Q. pseudosuber), as is pointed out by the accurate Ray, Hist. ii. p. 1387 (1688), who knew that tree in Italy, whereas he had only seen the acorns of the Vallonea at Venice, imported from Vallona.

The only spot in Italy where the Vallonea oak has any claim to be indigenous is near Tricase, in the extreme heel, where a fine group on the steep coast of the Adriatic has every appearance of being native, but as it is quite isolated, one cannot feel sure that it was not planted of old.

\section{XIV.-THE GENUS ROSMARINUS.}

\section{W. B. TurRILL.}

The genus Rosmarinus is limited to the Mediterranean Region, where it occurs in the soutbern parts of the European countries bordering this sea, in North Africa and as far east as Cyprus, the Troad, and also Cilicia, according to Boissier, Flora Orientalis, vol. iv. p. 636. Most systematists have limited the genus to one species, namely, Rosmarinus officinalis, L., although about twelve plants have been given specific rank by various authors. The following revision of the genus is based mainly on the excellent material preserved in the Kew Herbarium.

Rosmarinus, L., Gen. Pl., ed. v. p. 14, n. 38 (1754); Benth. Gen. et Spec. Labiat. p. 314 et in DC. Prod. vol xii. p. 360; Benth. et Hook. f., Gen. Plant., vol. ii. p. 1197 ; Briquet in Engler u. Prantl, Pflanzenfam., 3 A. p. 216.

Rosmarinus officinalis, L., Sp. Pl., ed. 1, p. 23 (1753); Briquet, Les Labiées des Alpes Maritimes, p. 179 (1891).

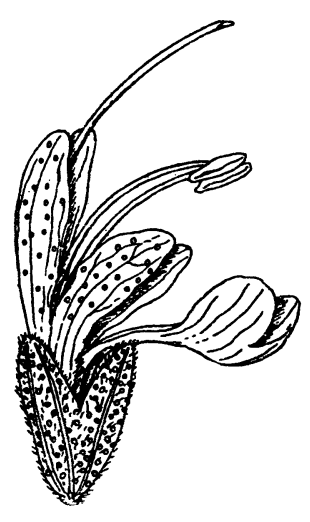

This species is the common rosemary and may be subdivided as follows :-

var. genuina, Turrill, var. nov. Frutex erectus, usque ad 6-10 dm. altus, ramosus. Folia linearia, saepissime circiter $2-3 \mathrm{~cm}$. longa, margine revoluta, subtus tomentosa. Inflorescentiae 1-2 cm. longae, pulverulentae vel subtomentosae vel subglabrae; bracteae lanceolatae vel lanceolato-ovatae; pedicelli circiter $3 \mathrm{~mm}$. longi. Calyx campanulatus, circiter $5 \mathrm{~mm}$. longus, glandulis sessilibus instructus, puberulus vel breviter tomentosus. Corolla circiter $1 \mathrm{~cm}$. longa vel major. 
Distr. Portugal, Spain, Balearic Islands, South France, Italy, Sicily, Malta, Dalmatia, Croatia, Istria, Switzerland, Greece, Crete (ex Halácsy, Conspect. Fl. Graec. vol. ii. p. 491), Macedonia (probably an escape from cultivation), Canaries, Azores, Madeira, Cyprus, Troad, Cilicia (ex Boissier, l.c.), Tunis, Egypt (ex Muschler, Man. Fl. Egypt, vol. ii. p. 829-var. pubescens, Pámp. ?).

forma erectus, Pasq. ex Bég. in Fiori e Paoletti, Fl. analit. d'Italia, vol. iii. p. 14 (1903). Suffrutex erectus.

Distr. As for the var. genuina.

forma humilis, Ten., Syl. Fl. Neapol. p. 16 (1831); forma procumbens, Pasq., Fl. Vesuviana, p. 79 (1869); var. prostratus, Pasq. in Cat. del Real Ort. Bot. di Napoli, p. 91 (1867); var. rupestris, Pasq. ex Bég. l.c. Suffrutex prostratus, ramis diffusis.

Distr. Italy, and probably elsewhere.

forma albiflorus, Bég. l.c. Corolla alba.

Distr. Italy, and probably elsewhere.

var. rigidus, Car. et Saint-Lag., Etude des Fleurs, p. 657 (1889); Rouy et Fouc., Fl. de France, xi. p. 249 (1909). R. rigidus, Jord. et Four., Brev. pl. nov., fasc. i. p. 43 (1866). Suffrutex robustus, caulibus rigidis, ramis erectis, foliis plus minusve erectis virentibus, corolla grandiuscula.

Distr. Southern France, Italy, Spain.

var. angustifolius, Guss., Syn. Fl. Siculae, vol. i. p. 20 (1842); $R$. angustifolius, Mill., Dict., ed. 8, no. 1 (1768); var. angustissimus, Fouc. et Mand. in Bull. Soc. Bot. Fr., vol. xlvii. p. 95 (1900); Rouy et Fouc. l.c. R. tenuifolius, Jord. et Four., 1.c. Suffrutex erectus, foliis patulis tenuibus circiter $1 \mathrm{~mm}$. latis obscure virentibus, corolla grandiuscula.

Distr. Southern France, Italy, Corsica.

var. latifolius, Bég. l.c. R. latifolius, Mill., l.c. no. 2 ; Rouy et Fouc. l.c. $R$. flexuosus, Jord. et Four., l.c. p. 44 . Suffrutex caulibus flexuosis, ramis patulis contortisque, foliis patulis latiusculis margine vix revolutis.

Distr. Southern France, Italy.

var. pubescens, Pamp. in Bull. Soc. Bot. It., 1914, p. 16 et Pl. Tripolit., p. 16 (1914). I have not seen this plant, but the original description is as follows: Inflorescentiae dense pubescentes, nec puberulae ut in typo et varietatibus nonnullis nec tomentosovillosae ut in var. lavandulaceo ( $R$. lavandulaceus).

Distr. Tripoli: Mesellata, Tarhuna, Gariau (ex Pampanini).

forma roseus, Pamp., l.c. Flores rosei.

Distr. Tripoli: Tarhuna (ex Pampanini).

Rosmarinus laxiflorus, de Noé in Balansa, Pl. d'Algerie, in 1852 , no. 443 (with printed Latin description); Lange, Pug., p. 178 (1863); Willkomm et Lange, Prod. Fl. Hisp. vol. ii. p. 419 (1870). R. officinalis, var. laxiflorus, Munby, Cat. Pl. Alg., 1859, p. 24; ed. 2, 1866, p. 27; Battandier et Trabut, Fl. de l'Algerie. 


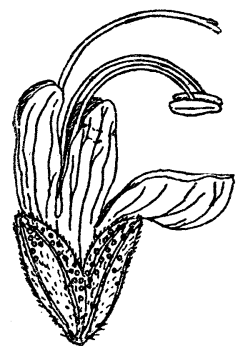

Suffrutex prostratus, ramis plus minusve contortis. Folia linearia, circiter $1.6 \mathrm{~cm}$. longa et $2 \mathrm{~mm}$. lata, margine revoluta. Inflorescentiae 2-3 cm. longae, laxiflorae; bracteae ovatae, apice acuminatae, $1.5 \mathrm{~mm}$. longae ; pedicelli $5 \mathrm{~mm}$. longi. Calyx campanulatus, $4 \mathrm{~mm}$. longus, leviter puberulus et glandulosus. Corolla 7-8 mm. longa.

Distr. Algeria: Santa Cruz, Oran; DjebelSantr, Oran.

Spain: Cordoba; Cartagena (ex Willkomm et Lange, l.c.).

Rosmarinus lavandulaceus, de Noé in Balansa, Pl. d'Algerie, 1852, no. 444 (with printed Latin description); Debeaux in Mém. Assoc. Franç. avanc. sci. Oran, 1888, p. 312. R. officınalis, var. lavandulaceus, Munby, Cat. Pl. Alg., 1859, o. 24; ed. 2, 1866, p. 27; Battandier et Trabut, l.c., p. 690 (1890); Debeaux, Fl. de la Kabylie, p. 293 (1894).

Suffrutex prostratus (?). Folia $1.5 \mathrm{~cm}$. longa vel minora, $1 \mathrm{~mm}$. lata, valde revoluta. Inflorescentiae compactae, multiflorae, $2 \cdot 5-3.5 \mathrm{~cm}$. longae; bracteae subrotundae, $2 \mathrm{~mm}$. longae, acutae; pedicelli usque ad $3 \mathrm{~mm}$. longi. Calyx tubulosus, $4 \mathrm{~mm}$. longus, dense albo-tomentosus et pilis longis tenuibus haud glandulosis instructus. Corolla $1 \mathrm{~cm}$. longa.

Distr. Algeria: plain of Andalous, near Oran; Cape Falcon, near Oran; Arzew, east of Oran; Colesh.

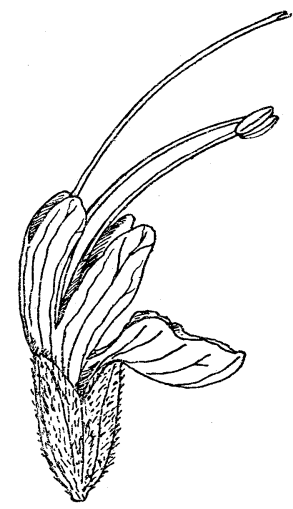

R. lavandulaceus.

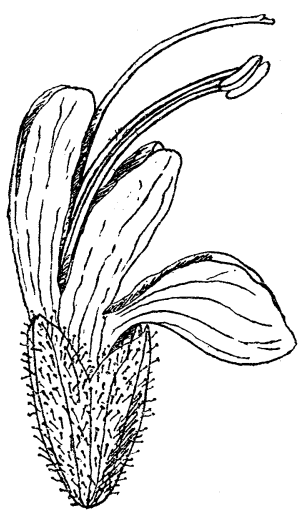

R. Tournefortii.

Rosmarinus Tournefortii, de Noé ex Battandier et Trabut, Fl. de l'Algerie, p. 690 (1890), nomen; $R$. officinalis, var. Tournefortii, de Noé in Billot flor. gall. et germ. exsic. no. 2124; Rosmarinus eriocalix, Jord. et Four., Brev. Pl. Nov., fasc. i. p. 44 (1866).

Suffrutex subprostratus, ramis contortis. Folia $1.5-2 \mathrm{~cm}$. longa, $2 \mathrm{~mm}$. lata, margine valde revoluta. Inflorescentiae usque ad $3.5 \mathrm{~cm}$. longae, breviter pubescentes et pilis longis apice glandulosis instructae; bracteae late ovatae, apice obtusae vel abrupte breviterque acuminatae, $3 \mathrm{~mm}$. longae; pedicelli usque ad $5 \mathrm{~mm}$. 
longi. Calyx tubulosus, $6 \mathrm{~mm}$. longus, breviter pubescens et pilis distinctis tenuibus glanduloso-capitatis praeditus. Corolla $1 \cdot 2 \mathrm{~cm}$. longa.

Distr. Algeria : Sidi-bel-Abbis, near Oran; Sehdon, near Oran; Mettili.

According to Captain Hilton-Simpson the Arabic name for this plant is Khlil.

\section{XV.-DECADES KEWENSES}

\section{Plantarum Novarum in Herbario Horti Regit \\ CONSERVATARUM.}

\section{DECAS XCVI.}

951. Miliusa dolichantha, $C r a i b$ [Anonaceae-Miliuseae]; a $M$. Roxburghiana, Hook. f. et Thoms., foliis subtus molliter pubescentibus, alabastris elongatis angustis distinguenda.

Ramuli primo densius brunneo- vel purpureo-brunneo-pubescentes, mox puberuli, demum glabri, cortice striato-reticulato cinereo obtecti. Folia plerumque oblonga vel oblongo-lanceolata, apice longius acuminata, acumine ipso acuto vel obtusiusculo, basi parum inaequilatera, cuneata vel rotundata, $6 \cdot 5-20 \mathrm{~cm}$. $\times 2 \cdot 8-7 \cdot 4 \mathrm{~cm}$., chartacea, supra in costa densius pubescentia, ceterum glabra, subtus praecipue in costa nervisque molliter pubescentia, nervis 9-12 supra inconspicuis subtus prominentibus, venis transversis longis subtus prominulis, petiolo circa $3 \mathrm{~mm}$. longo suffulta. Flores plerumque gemini, axillares (saepissime ex axillis foliorum delapsorum), pedicello et calyce et petalis externe ferrugineo-pubescentibus, pedicello 7-8 $\mathrm{mm}$. longo paulo supra medium bracteola $8 \mathrm{~mm}$. Ionga lineari-lanceolata basin versus bracteola minore instructo. Sepala aperta, anguste lanceolata, $1 \mathrm{~cm}$. longa, $1.5 \mathrm{~mm}$. lata, acuta. Petala exteriora sepalis similia nisi paulo breviora, interiora valvata, in alabastro vix 2.5 mm. longa. Stamina 6-7- seriata.

Eastern Himalaya. Abor Hills: Ringing and Rotung; $450 \mathrm{~m}$. ; flowers in December, Burkill 36606, 37593, 37674.

952. Vatica Shingkeng, Dunn [Dipterocarpaceae-Vaticeae]; $V$. lanceolatae, Blume affinis, sed foliis longe acuminatis et sepalis 2 auctis differt.

Arbor elata, glabra. Cortex crassus, griseo-brunneus, ramis lenticellatis. Folia alterna, lanceolata, longe acuminata vel caudata, basi rotundata, 9-18 cm. longa, chartacea, nervis 6-paribus ascendentibus trabeculis numerosis parallelis connexis subtus prominulis, petiolis $0 \cdot 6-1 \mathrm{~cm}$. longis. Flores ignoti. Capsula tarde dehiscens vel indehiscens, $2 \mathrm{~cm}$. longa, globosa, breviter acuminata, in calyce persistente aucto patente insidens, sepalis 2 exterioribus ovatis obtusis striatis $3 \mathrm{~cm}$. longis, interioribus 1-1.5 cm. longis. Semina angulata, pauca.

Eastern Himalaya. Abor Hills: Rengging to Janakmukh; 